\title{
Fenestrated total arch thoracic endovascular aortic repair: coming of age
}

\author{
George Joseph \\ Department of Cardiology, Christian Medical College, Vellore, India
}

Adv Interv Cardiol 2019; 15, 4 (58): 387-390

DOI: https://doi.org/10.5114/aic.2019.90325

Total aortic arch replacement is one of the most demanding of cardiovascular surgical procedures despite advances in related surgical technique and anesthesiology. In a recent retrospective study of 3265 patients who underwent open total arch replacement, in-hospital mortality at specialized centers was $10.8 \%$, and permanent neurologic deficit rate was 6.8\% [1]. Zone 0 hybrid arch exclusion has similar mortality $(10.3 \%$ vs. $10.2 \%)$ and permanent neurological deficit (8.9\% vs. $6.2 \%)$ as open total aortic arch replacement [2]. Given this situation, the less invasive alternative of thoracic endovascular aortic repair (TEVAR) seems alluring. But total arch TEVAR faces several challenges, the principal ones being preservation of flow into all the aortic arch branches despite covering their ostia with the arch endograft and avoiding intra-procedural cerebral embolization. Several other problems may be encountered during arch TEVAR, which may be anatomic (tortuous arch, type-3 arch, Gothic arch), hemodynamic (due to the force of left ventricular ejection), device-related (inability to conform to arch anatomy and material fatigue) and access-related (lack of suitable large-bore femoral access, iliac disease and aortic tortuosity) [3]. One or more of these factors may conspire to cause device delivery failure, malposition, malapposition and ultimately treatment failure.

Branched TEVAR, using inner branched devices, is one of two main methods of total arch endovascular repair [4]. The double inner branched arch endograft (Cook Medical, Bloomington, IN, USA) has been the most investigated; early results in 38 patients (technical success $84.2 \%$, 30-day mortality $13.2 \%$, cerebrovascular complications $15.8 \%$ ) were not optimal [5], but later outcomes with this device have been much better [6]. The technique requires a left carotid to left subclavian bypass to preserve flow into the latter, unless a three-branched version of the endograft is used [7]. There are other lim- itations: the device has to be custom made, does not appose the aortic wall in the branch-bearing section, has fixed orientation of branches, requires a right carotid or axillary cut-down for delivering the innominate stent, has its proximal sealing zone deep in the ascending aorta (where it may cover ostia of coronary bypass grafts) and the nose cone of the delivery system almost inevitably has to cross the aortic valve [8]. Outcomes obtained with another branched device designed for total arch TEVAR, the Relay double inner branched stent-graft (Terumo Aortic, Glasgow, UK), have been disappointing: initial results in 24 patients revealed $16.7 \%$ in-hospital mortality and $25 \%$ cerebrovascular event rate [9].

Fenestrated TEVAR is the other major method of total arch TEVAR. The main difficulty of fenestrated arch repair is accurate positioning of fenestrations; given the wide variation seen in aortic arch anatomy, customization to suit individual anatomy is necessary, and offthe-shelf devices are unlikely to work in a large segment of patients. In situ fenestration of arch endografts has been one way of dealing with this issue; however, when using this technique in total arch TEVAR, adjunctive procedures to maintain cerebral perfusion are required during the operation [10]. This technique could potentially result in fraying of fenestration edges and cause type-3 endoleak.

Another approach to fenestrated arch TEVAR has been the use of non-sealing fenestrations that are much larger than the target arch branches and allow some flexibility in endograft positioning; these endografts are custom-designed to suit the patient's arch anatomy and may have stent struts going across them. One example is the Najuta semi-custom thoracic fenestrated stent-graft (Kawasumi Laboratories, Tokyo, Japan), which is loaded in a pre-shaped sheath appropriate for the patient's aortic arch anatomy [11]. Another example

\section{Corresponding author:}

George Joseph MD, DM, Department of Cardiology, Christian Medical College, Vellore 632004, India, phone: +91 9894783163 ,

e-mail: joseph59@gmail.com

Received: 1.12.2019, accepted: 2.12.2019. 
is the homemade double-fenestrated stent-graft reported by Canaud et al. [12]. Deployment of covered stents through the fenestrations in these two devices will not produce local sealing or is not possible because of crossing stent struts; sealing is achieved by apposition of the endograft to the aortic wall, and the technique is thus appropriate only for distal arch or inner curve pathology.
Yet another approach to fenestrated total arch TEVAR is the use of physician-modified endografts with customized small (sealable) fenestrations and partial constraining of the endograft to enable manipulation of the endograft in the arch to align the fenestrations and to create a perigraft working space for cannulation of arch branches through the fenestrations. The technique of constrain-
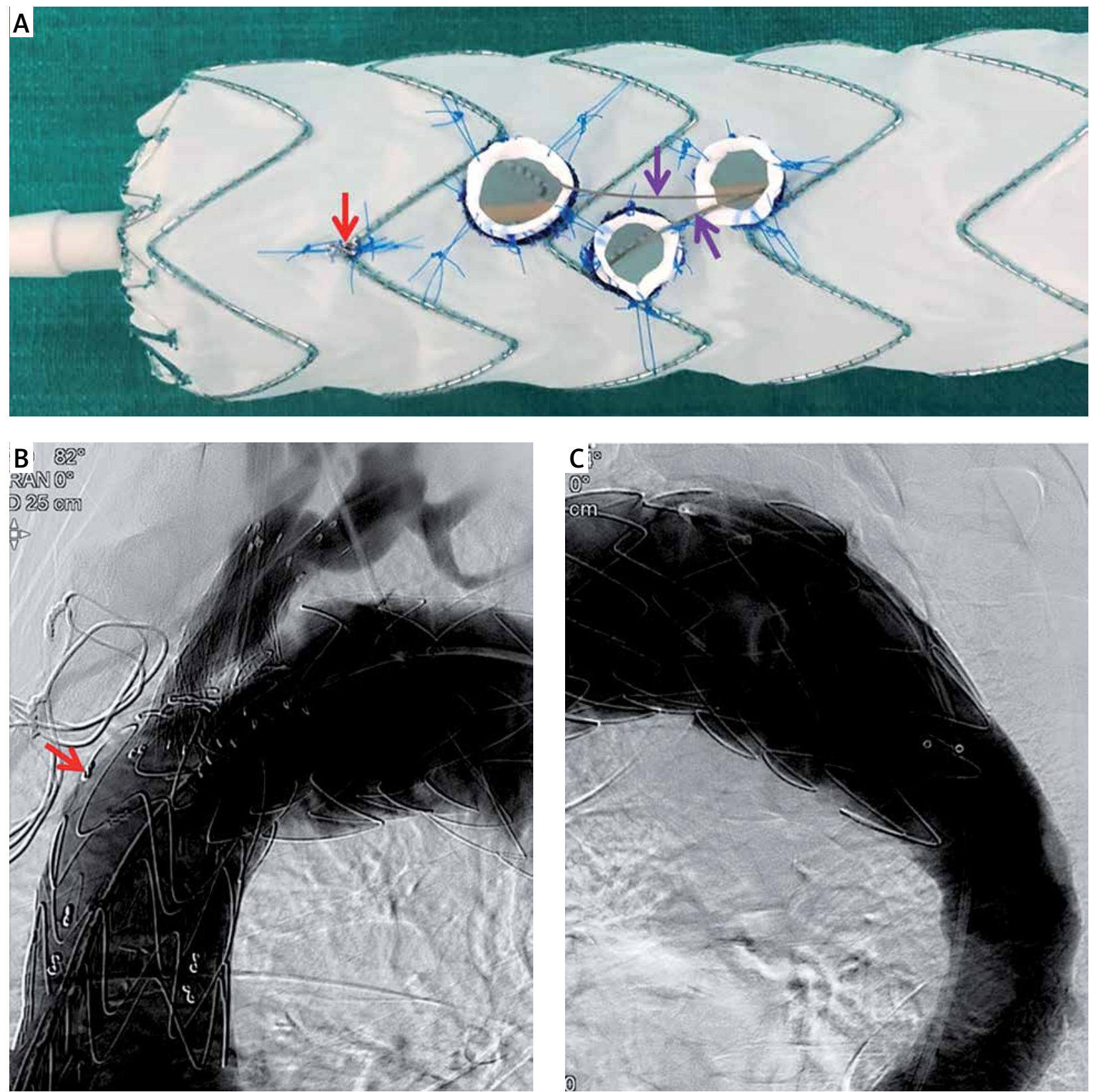

Figure 1. A - Valiant Captivia thoracic endograft with three fenestrations meant for the innominate, left common carotid and left subclavian arteries respectively (from left to right). Short cuffs made of vascular graft are attached to each fenestration to improve sealing. Two pre-cannulating nitinol guidewires (purple arrows) are seen emerging from the left subclavian artery fenestration and returning through the other two. A figure-of-8 radio-opaque marker (red arrow) is fixed on to the endograft at 12 o'clock position. B, C - Completion aortograms obtained after deployment of the above endograft in a patient with distal aortic arch aneurysm. The figure-of- 8 marker is seen on the outer curve of the endograft. Flow into the arch branches is preserved and there is no endoleak 
ing endografts using a longitudinal diameter-reducing wire was described by Oderich [13]. Recently Zhu et al. [14] reported use of this technique to constrain arch endografts with both small and oversized fenestrations in total arch endovascular repair. Their diameter-reducing wire was located directly opposite (180 degrees away) from the fenestrations on the endograft; with correct endograft deployment, the fenestrations would face the outer curve and the wire would be on the inner curve of the arch; however the splinting effect of this wire could limit the ability of the endograft to flex on the side of the lesser curve and potentially lead to malalignment. Having this wire run along the aspect of the arch endograft facing the outer curve is not appropriate as it would interfere with the fenestrations. In this issue of the "Advances in Interventional Cardiology" Kazimierczak et al. describe a novel technique of constraining arch endografts that overcomes the above problem by using dual lateral diameter-reducing wires located $90^{\circ}$ away from the fenestrations; besides allowing greater constraining of the endograft, this technique restricts flexion of the endograft to the plane of the arch which may help align the fenestrations better [15].

Manipulation of constrained endografts to align fenestrations in the aortic arch increases the risk of embolization, and therefore techniques that allow immediate and complete deployment of unconstrained fenestrated arch endografts in one go are preferable; the pre-requisite for this is a system for accurate positioning of fenestrations. Use of externalized guidewires that pre-cannulate fenestrations in unconstrained arch endografts is one approach to total arch TEVAR that enables immediate and complete endograft deployment and ensures easy access through fenestrations into target branches [16]. However, pre-cannulation of more than one fenestration with externalized guidewires is technically complex and limits its widespread use.

At our center we have moved away from externalizing pre-cannulating guidewires and have developed an alternative reliable and simple system for obtaining axial and rotational accuracy of fenestration position [4]. Customized, sealable fenestrations with robust, radiopaque edges are created for all three arch branches, based on computed tomography angiography data, on a Valiant Captivia thoracic endograft (Figure 1 A, Medtronic Vascular, Santa Rosa, CA, USA). A short cuff made of vascular graft is affixed to the fenestration edge to provide better sealing when required, as within an aneurysm zone or adjacent to an intimal tear. Cuffed fenestrations are pre-cannulated, but the wire is not externalized; instead, it loops back through another fenestration catheters can ride up these wires to cross the respective fenestrations after which a parallel guidewire can be passed through the catheter into the target arch branch. A radiopaque figure-of- 8 marker is fixed at the 12 o'clock position on the endograft and brought up just under the endograft cover during re-sheathing. Correct rotational orientation is obtained by appropriate rotation of the endograft delivery system as it rides up a stiff guidewire from the descending aorta into the arch: the figure-of-8 marker should be seen on edge at the outer curve of the arch when viewed fluoroscopically, orthogonal to the plane of the arch. Correct axial orientation is obtained by positioning the left carotid artery fenestration just ahead of a taut guidewire passing from the left carotid artery to the left femoral artery (this wire marks the posterior margin of the left carotid artery origin accurately). The endograft is not constrained in any way and once in position, it is fully deployed in one go (achieving maximal wall apposition immediately and trapping aortic debris underneath). Short self-expanding covered stents (Fluency; Bard Peripheral Vascular, Tempe, AZ, USA) are usually deployed within the fenestrations (Figures $1 \mathrm{~B}$, C). The technique is completely percutaneous, using only $4 \mathrm{Fr}$ or $6 \mathrm{Fr}$ arterial accesses other than the large endograft delivery access. The ease and success of the technique have convinced us that total arch TEVAR using fenestrated endografts is ready to replace open and hybrid arch surgery in most situations. Industrial manufacture of such customized fenestrated arch endografts would be the next logical step to enable widespread use of the technique.

\section{Conflict of interest}

The author declares no conflict of interest.

\section{References}

1. Keeling WB, Tian DH, Leshnower BG, et al. Safety of moderate hypothermia with antegrade cerebral perfusion in total aortic arch replacement. Ann Thorac Surg 2018; 105: 54-61.

2. Preventza O, Garcia A, Cooley DA, et al. Total aortic arch replacement: a comparative study of zone 0 hybrid arch exclusion versus traditional open repair. J Thorac Cardiovasc Surg 2015; 150 : 1591-8.

3. Makaloski V, Tsilimparis N, Rohlffs F, et al. Endovascular total arch replacement techniques and early results. Ann Cardiothorac Surg 2018; 7: 380-8.

4. Joseph G. Commentary. Treatment of ascending aortic pathology with arch endografts that extend proximally. J Endovasc Ther 2019; 26: 463-6.

5. Haulon S, Greenberg RK, Spear R, et al. Global experience with an inner branched arch endograft. J Thorac Cardiovasc Surg 2014; 148: 1709-16.

6. Spear R, Haulon S, Ohki T, et al. Subsequent results for arch aneurysm repair with inner branched endografts. Eur J Vasc Endovasc Surg 2016; 51: 380-5.

7. Spear R, Clough RE, Fabre D, et al. Total endovascular treatment of aortic arch disease using an arch endograft with 3 inner branches. J Endovasc Ther 2017; 24: 534-8.

8. Lioupis C, Corriveau MM, MacKenzie KS, et al. Treatment of aortic arch aneurysms with a modular transfemoral multibranched stent graft: initial experience. Eur J Vasc Endovasc Surg 2012; 43: 525-32. 
9. Ferrer C, Cao P, Coscarella C, et al. iTalian Reglstry of doUble inner branch stent graft for arch PatHology (the TRIUmPH Registry). J Vasc Surg 2019; 70: 672-82.e1.

10. Kopp R, Katada Y, Kondo S, et al. Multicenter analysis of endovascular aortic arch in situ stent graft fenestrations for aortic arch pathologies. Ann Vasc Surg 2019; 59: 36-47.

11. Fukushima S, Ohki T, Toya N, et al. Initial results of thoracic endovascular repair for uncomplicated type B aortic dissection involving the arch vessels using a semicustom-made thoracic fenestrated stent graft. J Vasc Surg 2019; 69: 1694-703.

12. Canaud L, Ozdemir BA, Chassin-Trubert L, et al. Double homemade fenestrated stent graft for total endovascular aortic arch repair. J Vasc Surg 2019; 70: 1031-8.

13. Oderich GS. Diameter-reducing wire to facilitate deployment of a modified Zenith fenestrated stent graft. Ann Vasc Surg 2010; 24: 980-4.

14. Zhu J, Dai X, Noiniyom P, et al. Fenestrated thoracic endovascular aortic repair using physician-modified stent grafts (PMSGs) in Zone 0 and Zone 1 for aortic arch diseases. Cardiovasc Intervent Radiol 2019; 42: 19-27.

15. Kazimierczak A, Rynio P, Jędrzejczak T, et al. Utility of dual longitudinal diameter-reducing ties in aortic arch thoracic endovascular aortic repair. Adv Interv Cardiol 2019; 15: 485-8.

16. Joseph G, Premkumar P, Thomson V, et al. Externalized guidewires to facilitate fenestrated endograft deployment in the aortic arch. J Endovasc Ther 2016; 23: 160-71. 